\title{
Cyclovoltammetrische Bestimmung redoxaktiver Gase mittels eines Festelektrolytsensors
}

\author{
M. Schelter ${ }^{1}$, J. Zosel ${ }^{1}$, W. Oelßner ${ }^{1}$, U. Guth ${ }^{1,2}$, M. Mertig ${ }^{1,2}$ \\ ${ }^{1}$ Kurt-Schwabe-Institut für Mess- und Sensortechnik e.V. Meinsberg, Deutschland \\ schelter@ksi-meinsberg.de \\ ${ }^{2}$ Professur für Physikalische Chemie, Mess- und Sensortechnik, Techn. Universität Dresden, Deutschland
}

\begin{abstract}
Kurzfassung
Systematische Untersuchungen zur Cyclovoltammetrie wurden an einem kommerziell verfügbaren Festelektrolytsensor im Durchflussmodus durchgeführt. Dabei wurden die Einflussgrößen Zelltemperatur, Elektrodengröße und Potentialvorschubgeschwindigkeit variiert und Bedingungen gefunden, bei denen es für $\mathrm{H}_{2^{-}}$, CObzw. $\mathrm{O}_{2}$-haltige Messgase zur Peakausbildung im Messsignal kommt. Im unteren Konzentrationsbereich gibt es für jedes dieser Gase einen linearen Zusammenhang zwischen der Konzentration und den auswertbaren Peakeigenschaften Höhe und Fläche. Für $\mathrm{H}_{2}$ ist das der Bereich bis $100 \mathrm{Vol}$.-ppm und für $\mathrm{CO}$ der bis 500 Vol.-ppm. Für $\mathrm{O}_{2}$ konnte die lineare Korrelation im gesamten untersuchten Konzentrationsbereich von 0 bis 500 Vol.-ppm gefunden werden. Trotz der katalytisch hochaktiven Platinelektroden kann beispielsweise $\mathrm{H}_{2}$ bei unterschiedlichen Beimengungen von $\mathrm{O}_{2}$ im Messgas selektiv erfasst werden.
\end{abstract}

Schlüsselwörter: Festelektrolytsensor, YSZ, Cyclovoltammetrie, Querempfindlichkeit, Selektivität

\section{Einleitung}

Festelektrolytsensoren mit Platinelektroden, die mit stationären Messmethoden wie Potentiometrie, Amperometrie oder Coulometrie betrieben werden, sind nicht dazu geeignet, gleichzeitig mehrere Analyte mit hinreichender Selektivität zu bestimmen. Beispielsweise stellt sich an einer Zelle des Typs „Messgas,Pt|YSZ|Pt,Luft“ eine Zellspannung entsprechend dem Gleichgewichts-Sauerstoffpartialdruck im Messgas ein. Dabei tragen alle im Messgas vorhandenen Spezies, die an der heißen Platinelektrode des Festelektrolytsensors mit Sauerstoff reagieren, zum Sauerstoffpartialdruck und folglich zur Potentialbildung bei. Aus der gemessenen Zellspannung kann demnach nicht auf Anzahl, Art und Konzentration der im Messgas enthaltenen Spezies geschlossen werden.

Im Gegensatz zu den oben genannten stationären Messmethoden besteht die Grundidee der hier vorgestellten Untersuchungen darin, durch Anwendung der Cyclovoltammetrie [1] die Selektivität von Festelektrolytsensoren zu erhöhen. Durch die periodische Variation der an den Sensor angelegten Zellspannung $U_{p}(t)$ wird unter Ausnutzung verschiedener Reaktionskinetiken und/oder unterschiedlicher Elektrodenpotentiale die zeitlich getrennte selektive Bestimmung mehrerer Analyte ermöglicht. Es wird gezeigt, dass mit dieser Methode Brenngase wie $\mathrm{H}_{2}$ auch bei einem Überschuss an $\mathrm{O}_{2}$ an katalytisch hochaktiven Elektroden bestimmt werden können.

\section{Methoden und Materialien}

Die in den vorgestellten Untersuchungen verwendete, in Abb. 1 dargestellte Messanordnung enthält eine beheizbare rohrförmige Zelle des Typs „Messgas,Pt|YSZ|Pt,Luft", durch die das Messgas mit Volumenströmen zwischen 5 und $50 \mathrm{~mL} / \mathrm{min}$ hindurchströmt [2].

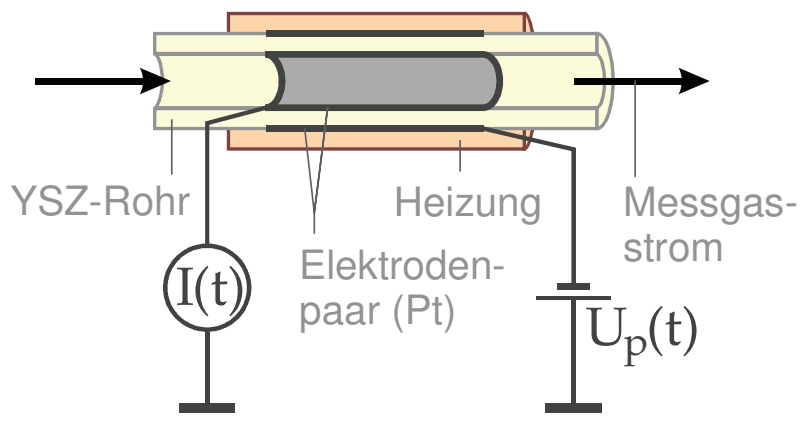

Abb. 1. Messanordnung des Festelektrolytsensors (Elektrodenflächen innen/außen: $7,5 \mathrm{~cm}^{2} / 11,3 \mathrm{~cm}^{2}$ ) mit cyclovoltammetrischer Messeinrichtung; $U_{p}(t)$ : periodisch variierte Zellspannung; $I(t)$ : Messsignal.

Die Zellspannung wurde von einem Messsystem Reference $600^{\mathrm{TM}}$ (Gamry Instruments, Warminster, USA) bereitgestellt, mit dem gleichzeitig das Stromsignal gemessen wurde. Die in dieser Arbeit verwendeten Gase $\mathrm{N}_{2}, 1001$ Vol.-ppm $\mathrm{H}_{2}$ in $\mathrm{N}_{2}$ und 1992 Vol.-ppm CO in $N_{2}$ (alle von Rießner-Gase $\mathrm{GmbH}$, Lichtenfels, Deutschland) wurden mittels Masseflussreglern (Brooks Instrument Company, Hatfield, USA) gemischt, wodurch definierte Prüfgaskonzentrationen resultierten. 


\section{Ergebnisse und Diskussion}

\section{I-U-Kennlinien von Festelektrolytsensoren}

Im Verlauf eines Cyclovoltammogramms ändern sich infolge der Potentialveränderung die Reaktionsmechanismen der Elektrodenprozesse. Für das Verständnis der komplexen Entwicklung dieser Prozesse ist es zweckmäßig, zunächst die in Abb. 2 gezeigte I-U-Kennlinie der Zelle mit getrenntem Gasraum des Typs „Stickstoff,Pt|YSZ|Pt, Luft" zu betrachten.

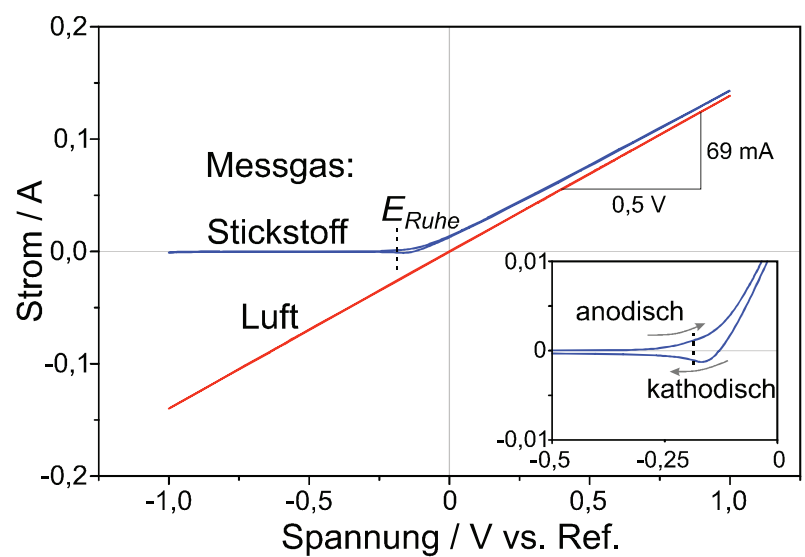

Abb. 2. I-U-Kennlinien des Festelektrolytsensors; Zelltemperatur: $750{ }^{\circ} \mathrm{C}$; Potentialvorschubgeschwindigkeit: $100 \mathrm{mV} \mathrm{s}^{-1}$; $\quad$ Messgasvolumenstrom: $10 \mathrm{~mL} \mathrm{~min}^{-1}$; Gleichgewichtspotential der Kurve in $\mathrm{N}_{2}$ : $-183,5 \mathrm{mV}$ (gestrichelte Linien); Inset: Vergrößerung der Kurve mit $\mathrm{N}_{2}$ als Messgas im Bereich von $-0,5$ bis $0 \mathrm{~V}$.

Bei positiver Polarisation der Messgaselektrode wird $\mathrm{O}_{2}$ von der Referenzelektrode durch den Festelektrolyt in den Messgasstrom gepumpt. Dabei wird Sauerstoff an der Kathode zu Oxidionen reduziert, diese durch die $\mathrm{O}^{2-}$-Leitfähigkeit zur Anode geleitet und dort $\mathrm{zu}_{2}$ oxidiert. Strombegrenzend wirkt in diesem Spannungsbereich hauptsächlich der Ohmsche Zellwiderstand, aus dem ein linear mit der Spannung ansteigender Stromfluss resultiert. Bei negativer Polarisation $\left(\mathrm{O}_{2}\right.$ wird aus dem Messgasstrom heraus und zur Referenzelektrode gepumpt) kann bei Abwesenheit von $\mathrm{O}_{2}$ im Messgas kein lonenstrom durch den Festelektrolyt fließen (blockierende Elektrode). Eine linear ansteigende Überspannung in die anodische Richtung resultiert gemäß der Butler-VolmerGleichung in einem exponentiell ansteigenden Stromfluss, der oberhalb von etwa $0 \mathrm{~V}$ in den oben beschriebenen linearen Bereich übergeht.

In kathodische Richtung befindet sich in der Nähe des Gleichgewichtspotentials ein Peak (siehe Inset in Abb 2). Es wird davon ausgegangen, dass dieser hauptsächlich auftritt, weil Sauerstoff, der zuvor bei positiven Spannungen in das Zellinnere gepumpt wurde, noch nicht vollständig mit dem Messgasstrom aus der Zelle ausgespült wurde. In diesem Fall würde der $\mathrm{O}_{2}$-Reduktionspeak bei dieser Zelle nicht wie sonst in der Voltammetrie durch Diffusionslimitierungen, sondern hauptsächlich durch Ausspülen von $\mathrm{O}_{2}$ mit dem Messgasstrom entstehen.

Weiterhin ist in Abb. 2 zum Vergleich die I-UKennlinie einer gassymmetrischen Festelektrolytzelle dargestellt (rote Gerade). Die hohe Sauerstoffverfügbarkeit im Messgas Luft bewirkt, dass auch bei hohen Überspannungen keine Diffusionslimitierungen zu beobachten sind. Durch die Gassymmetrie verläuft das Stromsignal punktsymmetrisch um den Koordinatenursprung, und das Gleichgewichtspotential der Messgaselektrode beträgt unter Vernachlässigung thermisch bedingter Asymmetriespannungen genau $0 \mathrm{~V}$ gegenüber der Referenzelektrode. Für den untersuchten Spannungsbereich ergibt sich ein linearer Stromverlauf, weil keine Diffusionslimitierungen an den Elektroden auftreten.

Der aus dem Anstieg des linearen Stromverlaufs berechnete Gesamtwiderstand der Zelle beträgt $R=7,2 \Omega$. Er repräsentiert die Summe aus den Polarisationswiderständen beider Elektroden sowie dem Elektrolytwiderstand. Der Elektrolytwiderstand lässt sich aus der ionischen Leitfähigkeit (nach [3]: $2,07 \mathrm{~S} \mathrm{~m}^{-1}$ ) sowie den Abmessungen des Festelektrolyts $\left(A=8,72 \mathrm{~cm}^{2}, d=1,0 \mathrm{~mm}\right)$ zu $0,55 \Omega$ berechnen. Der Polarisationswiderstand beider Elektroden beträgt demnach jeweils $28 \Omega \mathrm{cm}^{2}$.

Für weitere Untersuchungen an Zellen mit getrennten Gasräumen ist der negative Spannungsbereich von Interesse, in dem die Oxidations- und Reduktionsprozesse der hier interessierenden Analyte wie Wasserstoff und Sauerstoff an der MessgasElektrode dominieren.

\section{Peakausbildung bei redoxaktiven Substanzen}

Mittels der Cyclovoltammetrie wurden Versuchsbedingungen gesucht, bei denen es für stickstoffhaltige Messgase, die Spuren von $\mathrm{H}_{2}, \mathrm{O}_{2}$ oder $\mathrm{H}_{2} \mathrm{O}$ enthalten, zu den klassischen Zusammenhängen der Voltammetrie bezüglich der Peakhöhe kommt. Dazu wurden die Zelltemperatur, die Elektrodenfläche sowie die Potentialvorschubgeschwindigkeit variiert. Die Ergebnisse der Untersuchungen werden im Folgenden erläutert.

Die bei unterschiedlichen Zelltemperaturen aufgenommenen Cyclovoltammogramme $\mathrm{O}_{2}$-haltiger Messgase sind in Abb. 3 abgebildet. Erwartungsgemäß wird der in kathodische Richtung auftretende Sauerstoff-Reduktionspeak mit steigender Temperatur größer, und das Maximum verschiebt sich zu positiveren Spannungen. Der Peak erreicht bei Temperaturen oberhalb von $600{ }^{\circ} \mathrm{C}$ eine Fläche, die eine quantitative Auswertung ermöglicht.

Die in Abb. 4 dargestellten Voltammogramme bei unterschiedlichen Elektrodengrößen belegen, dass sich die Elektrodenfläche auf die Hysterese des Stromes zwischen kathodischer und anodischer 
Richtung auswirkt. Je größer die Elektrode, desto größer ist der kapazitive Strom, der für die Umladung der Elektroden und Ausbildung von elektrochemischen Doppelschichten sorgt. Für cyclovoltammetrische Untersuchungen sind deshalb möglichst kleine Elektroden zu nutzen, um solche bei analytischen Applikationen unerwünschte kapazitive Ströme gering zu halten.

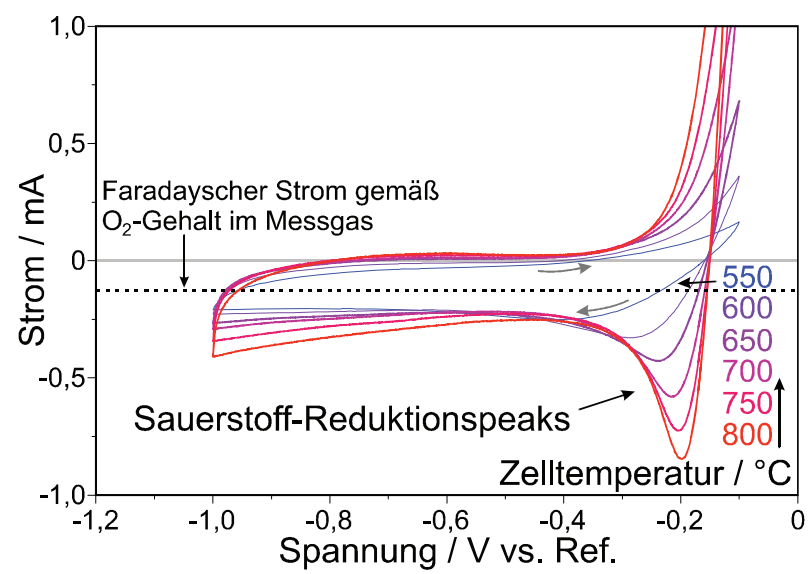

Abb. 3. Cyclovoltammogramme zur Variation der Zelltemperatur; Messgas: 40 Vol.-ppm $\mathrm{O}_{2}$ in $\mathrm{N}_{2}$; Messgasvolumenstrom: $10 \mathrm{~mL} \mathrm{~min}^{-1}$; Elektrodenfläche: $0,75 \mathrm{~cm}^{2}$; Ruhepotential: $-150 \mathrm{mV}\left(550^{\circ} \mathrm{C}\right)$ bis $-200 \mathrm{mV}\left(800^{\circ} \mathrm{C}\right)$; Potentialvorschubgeschwindigkeit: $1 \mathrm{~V} \mathrm{~s}^{-1}$; Startpotential: $-0,17 \mathrm{~V}$; dargestellt ist jeweils der zweite Zyklus.

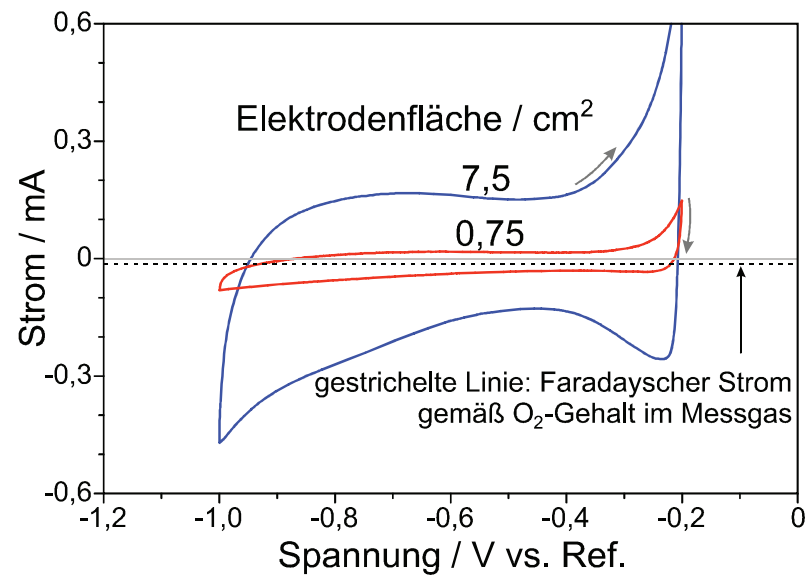

Abb. 4. Cyclovoltammogramme zur Variation der Elektrodengröße; Messgas: $5 \mathrm{Vol}$.-ppm $\mathrm{O}_{2}$ in $\mathrm{N}_{2}$; Messgasvolumenstrom: $10 \mathrm{~mL} \mathrm{~min}{ }^{-1}$; Zelltemperatur: $750{ }^{\circ} \mathrm{C}$; Ruhepotential: $-235 \mathrm{mV}$; Potentialvorschubgeschwindigkeit: $200 \mathrm{mV} \mathrm{s}^{-1}$; Startpotential: $-0,20 \mathrm{~V}$; dargestellt ist jeweils der zweite Zyklus.

Bei der klassischen Voltammetrie an Zellen mit wässrigem Elektrolyt steigt die Höhe eines Peaks im Voltammogramm gemäß der Randles-SevčikGleichung proportional zur Wurzel der Potentialvorschubgeschwindigkeit $\mathrm{dU}_{\mathrm{p}} / \mathrm{dt}$. Es wurde untersucht, ob sich dieser Zusammenhang an Festelektrolytzellen auf gleiche Weise einstellt. Abb. 5 zeigt Cyclovoltammogramme, die bei unterschiedlichen $\mathrm{dU}_{\mathrm{p}} / \mathrm{dt}$ an einer Zelle des Typs ${ }_{\text {, }} \mathrm{H}_{2}, \mathrm{H}_{2} \mathrm{O}, \mathrm{N}_{2}, \mathrm{Pt}|\mathrm{YSZ}|$ Pt,Luft" aufgenommenen wurden.

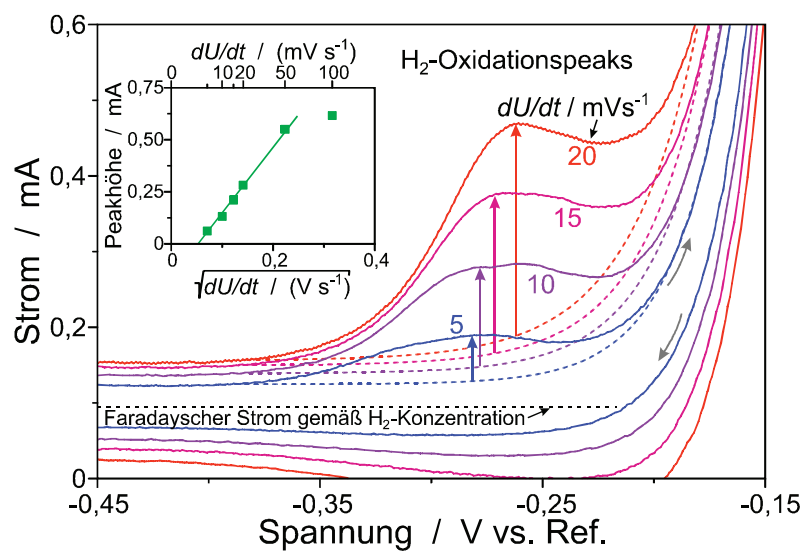

Abb. 5. Cyclovoltammogramme zur Variation der Potentialvorschubgeschwindigkeit; die Basislinien (gestrichelt, farbig) sind die exponentiellen Stromanstiege, die den bei wachsenden Spannungen zunehmenden Sauerstoff-Pumpströmen entsprechen, die Pfeillängen entsprechen den jeweiligen Höhen der $\mathrm{H}_{2}$-Oxidationspeaks; Inset: Peakhöhen der $\mathrm{H}_{2}$-Oxidationspeaks in Abhängigkeit der Wurzel der Potentialvorschubgeschwindigkeit; zusätzlich zu denen der Hauptabbildung sind Daten von 50 und $100 \mathrm{mV} \mathrm{s}^{-1}$ enthalten; Elektrodenfläche: $7,5 \mathrm{~cm}^{2}$; Zelltemperatur: $800{ }^{\circ} \mathrm{C}$; Messgas: $66 \mathrm{Vol} .-\mathrm{ppm} \mathrm{H}_{2}+$ $34 \mathrm{Vol}$-ppm $\mathrm{H}_{2} \mathrm{O}$ in $\mathrm{N}_{2}$; Messgasvolumenstrom:

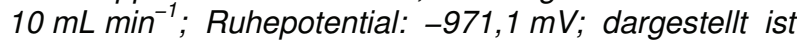
jeweils der zweite Zyklus.

Trotz dass im gesamten untersuchten Potentialbereich nahezu vollständiger Umsatz des Wasserstoffs gemäß dem Faradayschen Gesetz auftritt, kommt es zur zusätzlichen Ausbildung von $\mathrm{H}_{2}$ Oxidationspeaks im Bereich zwischen $-0,35$ und $-0,2$ V. Diese Peaks treten als Schulter im exponentiell anwachsenden $\mathrm{O}_{2}$-Evolutionsstrom (in Abb. 5 gestrichelt dargestellt) auf. Zur Auswertung der Peakhöhen wurde jeweils der $\mathrm{O}_{2}$-EvolutionsStrom als Basislinie vom Messsignal subtrahiert.

Bis zu dU $/ \mathrm{dt}=50 \mathrm{mV} \mathrm{s}^{-1}$ nimmt hier die Peakhöhe linear mit der Wurzel der Potentialvorschubgeschwindigkeit zu, wie im Inset von Abb. 5 dargestellt ist; allerdings verläuft diese Gerade aus noch unbekannter Ursache nicht durch den Koordinatenursprung.

Bei Potentialvorschubgeschwindigkeiten oberhalb von $50 \mathrm{mV} \mathrm{s}^{-1}$ gehen die $\mathrm{H}_{2}$-Oxidationspeaks in den exponentiellen Stromanstieg der $\mathrm{O}_{2}$-Evolution über, was mit einer geringeren Zunahme der auswertbaren Peakfläche verbunden ist und in einem Knick in der Auftragung der Peakhöhe in Abhängigkeit von der Wurzel der Potentialvorschubgeschwindigkeit resultiert.

Aus den erzielten Messdaten zur Variation von $\mathrm{dU}_{\mathrm{p}} / \mathrm{dt}$ lässt sich schlussfolgern, dass die klassischen Zusammenhänge der Voltammetrie auch bei Festelektrolytzellen gelten, sogar für vergleichsweise große Elektroden. 


\section{Sensitivität für oxidierbare und reduzierbare Gase}

Die Sensitivität des cyclovoltammetrisch betriebenen Festelektrolytsensors wurde für $\mathrm{H}_{2}, \mathrm{CO}$ und $\mathrm{O}_{2}$ unter Anwendung der gefundenen optimierten Versuchsbedingungen untersucht.

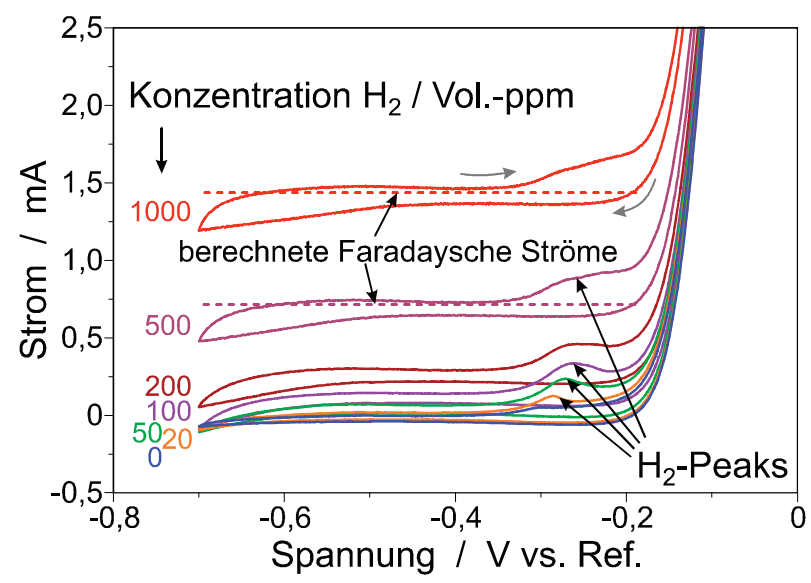

Abb. 6. Cyclovoltammogramme an Messgasen unterschiedlicher $\mathrm{H}_{2}$-Konzentrationen; Zelltemperatur: $750{ }^{\circ} \mathrm{C}$, Messgasvolumenstrom: $10 \mathrm{~mL} \mathrm{~min}^{-1}$, Elektrodenfläche: $7,5 \mathrm{~cm}^{2}$, Potentialvorschubgeschwindigkeit: $10 \mathrm{mV} \mathrm{s}^{-1}$.

Cyclovoltammogramme von Messgasen unterschiedlicher $\mathrm{H}_{2}$-Konzentration sind in Abb. 6 dargestellt. Die Peakauswertung erfolgte wie oben beschrieben durch Subtraktion der exponentiell ansteigenden Basislinie. Die in Abb. 7 gezeigten resultierenden Peakdaten zeigen, dass Peakhöhe und -fläche bis zu einer $\mathrm{H}_{2}$-Konzentration von etwa $100 \mathrm{Vol}$.-ppm linear wachsen und oberhalb dieser Konzentration nicht mehr wesentlich größer werden, da der Peak zunehmend in den exponentiellen Stromanstieg der $\mathrm{O}_{2}$-Evolution übergeht.

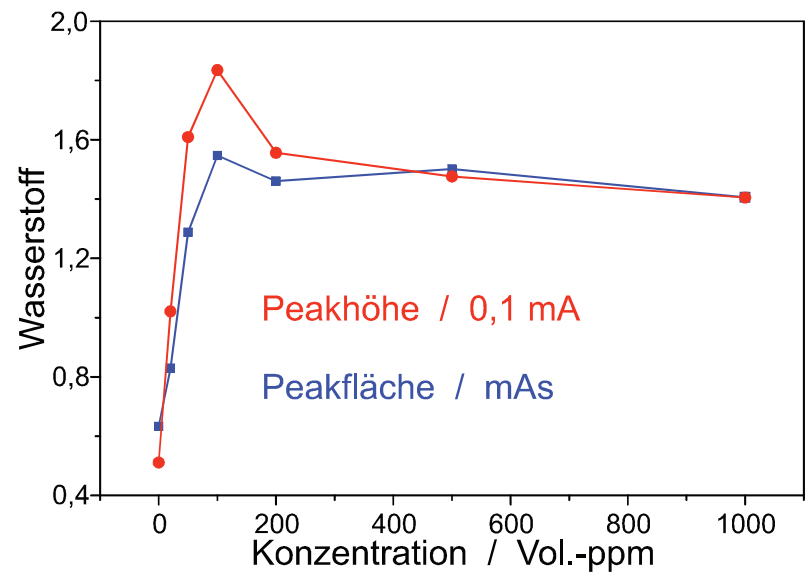

Abb. 7. Höhen und Flächen der in Abb. 6 gezeigten $\mathrm{H}_{2-}$ Peaks.

Bei den Voltammogrammen fällt auf, dass eine Änderung der Konzentration eine Parallelverschiebung der Kurven in Richtung der Stromachse bewirkt: je höher die Konzentration, desto größere Ströme fließen im gesamten untersuchten Potentialbereich. Hier wird der dynamische, potentialabhängige Strom von einem Faradayschen, potenti- alunabhängigen Stromfluss überlagert. Für 500 und 1000 Vol.-ppm sind die berechneten Faradayschen Ströme eingezeichnet, sie liegen genau im Strombereich der jeweiligen gemessenen Voltammogramme. Möglich wird dieser Faradaysche Umsatz durch die vergleichsweise großen Elektrodenflächen, durch den kontinuierlichen Zufluss von mit $\mathrm{H}_{2}$ beladenem Messgas und dadurch, dass im hier gezeigten Potentialbereich $\mathrm{H}_{2}$ immer zu einem wesentlichen Anteil oxidiert wird.

Neben wasserstoffhaltigen Messgasen wurden außerdem CO-haltige Messgase untersucht. Die resultierenden, in Abb. 8 gezeigten Cyclovoltammogramme belegen, dass mit der verwendeten Festelektrolytzelle auch Kohlenmonoxid voltammetrisch bestimmt werden kann. Abhängig von der Konzentration ändern sich auch hier sowohl die Größe des CO-Oxidationspeaks, als auch die Gesamtlage der Strom-Kurve. Die Gründe für die Parallelverschiebung der Strom-Spannungs-Kurven entlang der Stromachse sind wie bei Wasserstoff der Faradaysche Umsatz des ständig zuflieBenden CO-Anteils des Messgases im gewählten Potentialbereich oberhalb des Gleichgewichtspotentials. Die CO-Oxidationspeaks müssten gemäß den durch die Nernst-Gleichung vorgegebenen Gleichgewichts-Potentialen theoretisch bei deutlich negativeren Spannungen liegen. Sie finden sich allerdings wie die $\mathrm{H}_{2}$-Oxidationspeaks im Bereich von $-0,35$ bis $-0,2 \mathrm{~V}$. Es wird vermutet, dass zunächst im kathodischen Verlauf geleerte Sauerstoffdepots wieder gefüllt werden, bevor Substanzen an der Elektrode oxidiert werden können.

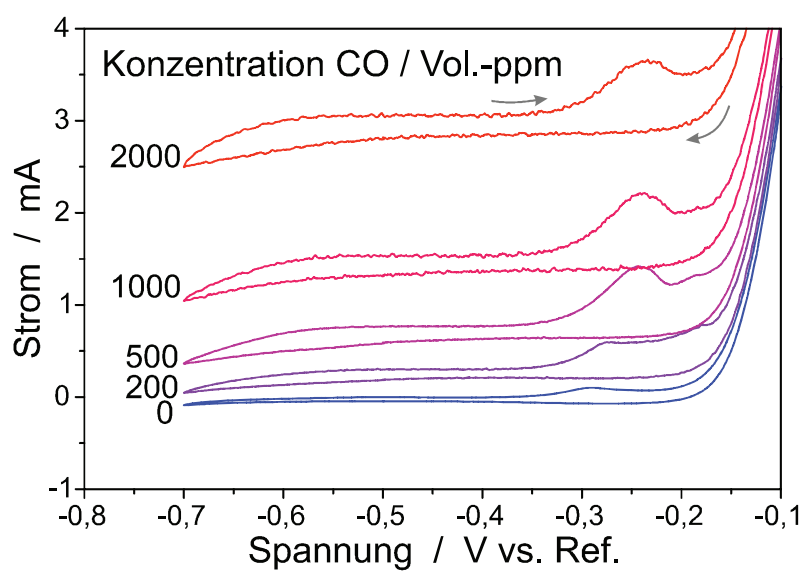

Abb. 8. Cyclovoltammogramme an Messgasen unterschiedlicher CO-Konzentrationen, Messgasvolumen-

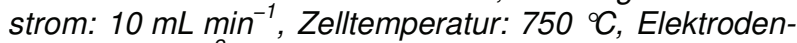
fläche: 7,5 cm $\mathrm{cm}^{2}$, Ruhepotentiale: $-221 \mathrm{mV}$ (0 Vol.-ppm CO) bis $-1106 \mathrm{mV}$ (2000 Vol.-ppm CO); Potentialvorschubgeschwindigkeit: $10 \mathrm{mV} \mathrm{s}^{-1}$; dargestellt ist jeweils der zweite Zyklus.

Für die Peakflächen und -höhen ergeben sich die in Abb. 9 gezeigten Abhängigkeiten von der COKonzentration. Zur Ermittlung dieser Daten wurde auch hier der exponentiell ansteigende $\mathrm{O}_{2}$-Pumpstrom als Basislinie herangezogen. Im Konzentra- 
tionsbereich bis 500 Vol.-ppm zeigt sich ein linearer Anstieg sowohl der Fläche als auch der Höhe. Oberhalb dieses Bereiches wachsen diese Parameter nicht weiter an, sie fallen sogar mit weiter steigender Konzentration leicht ab. Verglichen mit $\mathrm{H}_{2}$ kann im Fall von CO-haltigen Messgasen ein breiterer linearer Bereich für quantitative Bestimmungen genutzt werden.

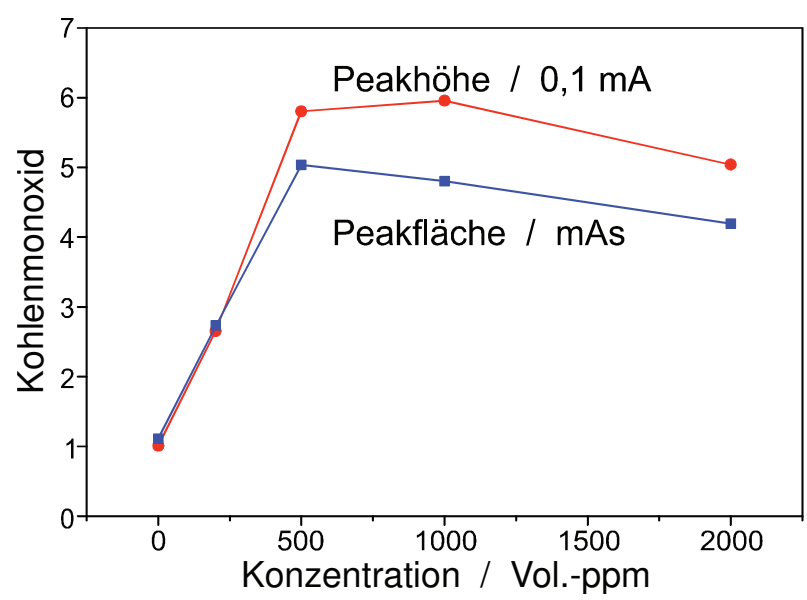

Abb. 9. Flächen und Höhen der CO-Peaks in den Cyclovoltammogrammen von Abb. 8.

An Messgasen mit unterschiedlichen $\mathrm{O}_{2}-$ Konzentrationen wurden die in Abb. 10 gezeigten Cyclovoltammogramme aufgenommen. Daraus wird ersichtlich, dass erwartungsgemäß auch bei $\mathrm{O}_{2}$ eine Konzentrationsänderung eine Verschiebung der Messkurven entlang der Stromachse bewirkt, allerdings in die entgegengesetzte Richtung wie bei Brenngasen. Peakhöhen wurden hier durch Differenzbildung zwischen den jeweiligen Absolutströmen am Peakminimum und bei $-0,55 \mathrm{~V}$ ermittelt.

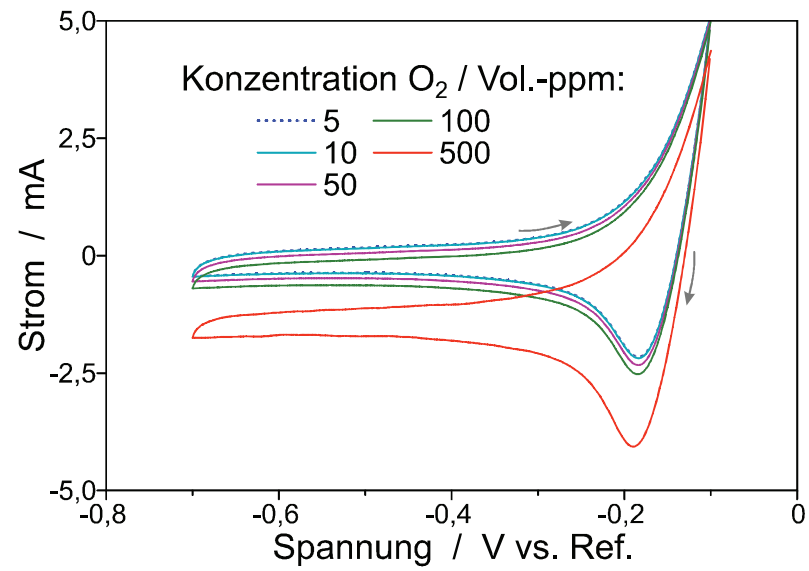

Abb. 10. Cyclovoltammogramme an Messgasen unterschiedlicher $\mathrm{O}_{2}$-Konzentrationen; Potentialvorschubgeschwindigkeit: $200 \mathrm{mV} \mathrm{s}^{-1}$; Elektrodenfläche: $7,5 \mathrm{~cm}^{2}$; Ruhepotentiale: $-241 \mathrm{mV}$ (5 Vol.-ppm $\mathrm{O}_{2}$ ) bis $-135 \mathrm{mV}$ (500 Vol.-ppm $\mathrm{O}_{2}$ ); Messgasvolumenstrom: $10 \mathrm{~mL} \mathrm{~min}^{-1}$; Zelltemp.: $750{ }^{\circ} \mathrm{C}$; dargestellt ist jeweils der 2. Zyklus.

Aus den Kalibrierdaten, die in Abb. 11 gezeigt sind, geht eine lineare Änderung der Höhe des $\mathrm{O}_{2-}$ Reduktionspeaks mit der Konzentration hervor. Die Ausgleichsgerade dieser Kalibrierung verläuft nicht durch den Koordinatenursprung, da während jedes Messzyklus zusätzlicher Sauerstoff ins Messgas gepumpt wird (bei Potentialen oberhalb von etwa $-0,15 \mathrm{~V}$; siehe Abschnitt „Peakausbildung bei redoxaktiven Substanzen"). Die Kalibrierergebnisse belegen die zuverlässige Bestimmbarkeit relativ kleiner $\mathrm{O}_{2}$-Konzentrationen im Messgas trotz der deutlich höheren $\mathrm{O}_{2}$-Mengen, die während der Messung ins Messgas gepumpt werden.

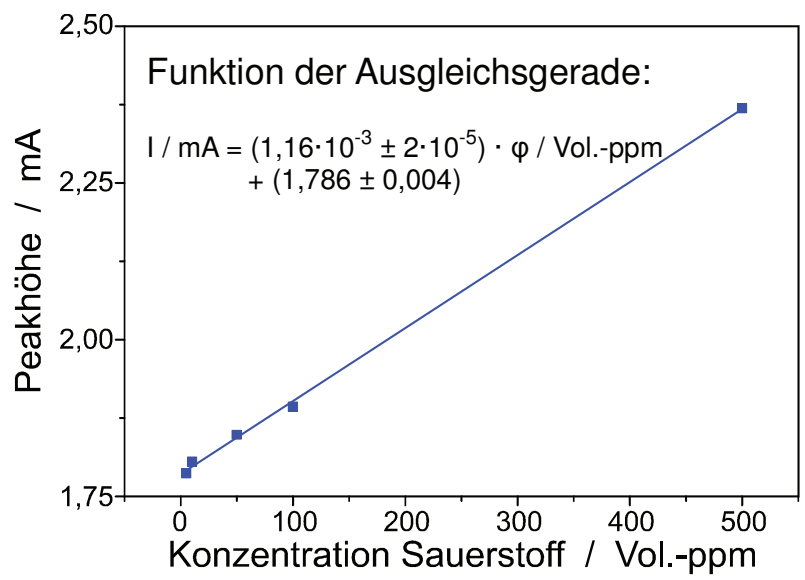

Abb. 11. Höhen der $\mathrm{O}_{2}$-Peaks in den Cyclovoltammogrammen von Abb. 10; die Peakhöhen wurden durch Differenzbildung zwischen den jeweiligen Absolutströmen am Peakminimum und bei -0,55 V ermittelt.

\section{Querempfindlichkeiten}

Abb. 12 zeigt Voltammogramme von Gasgemischen, die bei sehr unterschiedlichen $\mathrm{O}_{2}$-Konzentrationen im Bereich von 20 bis $>10^{4}$ Vol.-ppm jeweils 200 Vol.-ppm $\mathrm{H}_{2}$ enthalten.

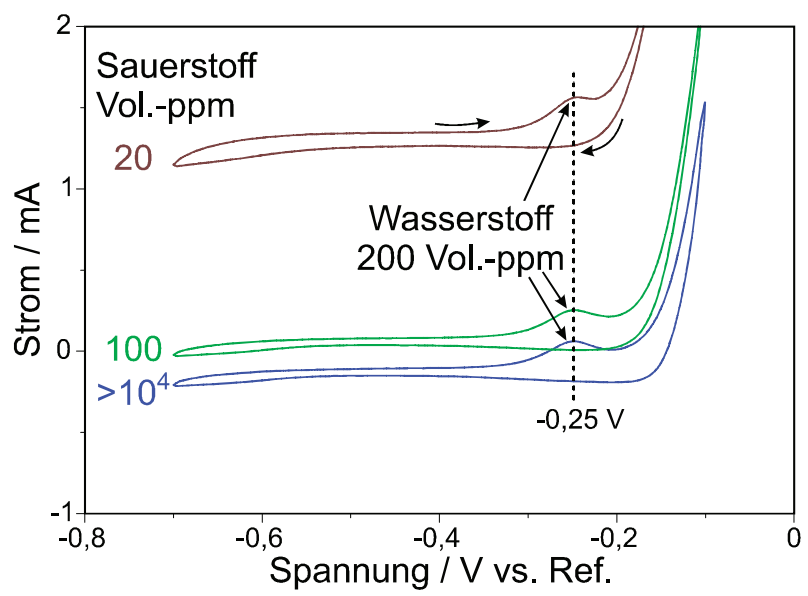

Abb. 12. Cyclovoltammogramme an Messgasen mit jeweils $200 \mathrm{Vol}$-ppm $\mathrm{H}_{2}$ und unterschiedlicher $\mathrm{O}_{2}$-Konzentrationen; Zelltemperatur: $700{ }^{\circ} \mathrm{C}$; Potentialvorschubgeschwindigkeit: $10 \mathrm{mV} / \mathrm{s}$; Messgasvolumenströme: 5 bis $50 \mathrm{~mL} / \mathrm{min}$; Elektrodenfläche: $7,5 \mathrm{~cm}^{2}$; Ruhepotentiale: $-161 \mathrm{mV}\left(>10^{4} \mathrm{Vol}\right.$-ppm $\left.\mathrm{O}_{2}\right)$ bis $-1030 \mathrm{mV}(20 \mathrm{Vol}$.ppm $\mathrm{O}_{2}$ ); dargestellt ist jeweils der zweite Zyklus.

Der unterschiedliche Sauerstoffgehalt wirkt sich hier zwar auf die Höhe des Grundstromes, nicht aber auf die Höhe der interessierenden $\mathrm{H}_{2}$ Oxidationspeaks und deren Potential aus, das 
jeweils bei ca. $-0,25 \mathrm{~V}$ liegt. Ursache für den mit steigender $\mathrm{O}_{2}$-Konzentration abnehmenden Grundstrom ist das wachsende Verhältnis von reduzierbaren zu oxidierbaren Bestandteilen, die pro Zeiteinheit an die Messgaselektrode gelangen. Aus Abb. 12 ist klar ersichtlich, dass mit cyclovoltammetrisch betriebenen Festelektrolytzellen beispielsweise in einem $\mathrm{H}_{2} / \mathrm{O}_{2}$-Gasgemisch Wasserstoff auch bei einem signifikanten Überschuss an $\mathrm{O}_{2}$ mit hoher Selektivität bestimmt werden kann.

\section{Zusammenfassung}

Systematische Untersuchungen zur Voltammetrie wurden an einem kommerziell verfügbaren Festelektrolytsensor im Durchflussmodus durchgeführt. Bei cyclovoltammetrischen Experimenten wurden die Einflussgrößen Zelltemperatur, ElektrodengröBe und Potentialvorschubgeschwindigkeit variiert und infolge Bedingungen gefunden, bei denen es für alle verwendeten Messgase zur Peakausbildung im Messsignal kommt. Diese Peakausbildung findet bei Brenngasen trotz der Tatsache statt, dass im gesamten untersuchten Potentialbereich nahezu vollständiger Analytumsatz gemäß dem Faradayschen Gesetz auftritt. Die Ursache für die zusätzliche Peakausbildung wird in einer teilweisen Verzögerung der Oxidationsprozesse vermutet, die durch Sauerstoffdefizite nahe der Elektrode hervorgerufen wird. Es wird angenommen, dass sich Sauerstoffdepots in Elektrodennähe befinden, die im kathodischen Verlauf geleert und im anodischen zunächst wieder gefüllt werden, bevor Substanzen an der Elektrode oxidiert werden können.

Im unteren Konzentrationsbereich gibt es für jedes der untersuchten Gase einen linearen Zusammenhang zwischen der Konzentration und den auswertbaren Peakeigenschaften Höhe und Fläche. Für $\mathrm{H}_{2}$ ist das der Bereich bis 100 Vol.-ppm und für $\mathrm{CO}$ der bis 500 Vol.-ppm. Für $\mathrm{O}_{2}$ konnte die lineare Korrelation im gesamten untersuchten Konzentrationsbereich von 0 bis $500 \mathrm{Vol}$-ppm gefunden werden. Trotz der katalytisch hochaktiven Platinelektroden kann mittels cyclovoltammetrisch betriebener Festelektrolytzellen beispielsweise Wasserstoff auch bei einem signifikanten Überschuss an $\mathrm{O}_{2}$ mit hoher Selektivität bestimmt werden.

\section{Literatur}

[1] G. Henze, Methoden. In: Polarographie und Voltammetrie: Grundlagen und analytische Praxis, Springer, Berlin, 22-65 (2001)

[2] M. Schelter, J. Zosel, W. Oelßner, U. Guth, M. Mertig, A solid electrolyte sensor for trace gas analysis, Sensors and Actuators B: Chemical 187, 209-214 (2012); doi: 10.1016/j.snb.2012.10.111

[3] J.-H. Park, R. N. Blumenthal, Electronic Transport in 8 Mole Percent Y2O3-ZrO2, Journal of the Electrochemical Society 136, 2867-2876 (1989); doi: 10.1149/1.2096302.

\section{Danksagung}

Die Untersuchungen wurden teilweise im Rahmen der mit Mitteln der Bundesministerien für Umwelt, Naturschutz und Reaktorsicherheit (BMU) sowie für Ernährung, Landwirtschaft und Verbraucherschutz (BMELV) geförderten Forschungsprojekte VESBA (FK 03KB045A) bzw. LIMOS (FK 22011110) durchgeführt. Die Verantwortung für den Inhalt dieser Arbeit liegt bei den Autoren, die für die Förderung danken. 